\title{
Editorial
}

\section{Special issue on wireless sensor networks: from theory to practice}

Wireless sensor network (WSN) is characterized by the dense deployment of sensor nodes that continuously observe physical phenomenon. The main advantages of WSN include its low cost, rapid deployment, self-organization, and fault tolerance. WSN has received tremendous interests of various research communities, and significant progresses have been made in various aspects including sensor platform development, wireless communication and networking, signal and information processing, as well as network performance evaluation and design. Such work has established good foundation for the studies in WSNs.

The research and development of WSNs are stimulated by novel exciting applications. For example, WSNs, particularly the wireless body sensor networks, make the old people/patient healthcare monitoring possible in smart home environments. Such WSNs pose significant technological challenges on application-specific signal and information processing algorithms having the severe network resource limitations such as node energy and wireless bandwidth. As a result, the application algorithms should be resource constrained and the network resource management should be application-driven, usually across multiple sensors and/or multiple network layers. To be scalable, such algorithms and protocols should also be distributed in nature via node local processing and collaboration with limited information exchange to achieve complex overall network goal.

This special issue is targeting on novel WSN applications and application-driven research and development to address the above challenges. Totally 17 papers were selected from numerous submissions after carefully reviews, and are divided into 3 categories.

The first category consists of 4 papers and is devoted to novel WSN applications. Shaofeng Wang et al. describe a novel ubiquitous healthcare system, uCare, which is designed for cardiovascular disease (CVD) examination and management. The paper presents the details regarding the system architecture, the real-time activity classification, ECG signal processing, and context-aware data fusion of uCare. By associating with the context information such as running, walking or sitting, the system can provide more precise and valuable information on vital signal analysis in human healthcare. Zhiqiang Zhang et al. develop a novel ubiquitous upper limb motion estimation method by fusion of measurements from wireless triaxial angular rate sensors, accelerometers, and magnetometers. The method is established on the structural movement relationship between the upper arm and the forearm, and employs an unscented Kalman filter for estimating the motion parameters. Andrei Tolstikov et al. compare two powerful fusion methods, dynamic Bayesian networks and Dempster-Shafer theory, for WSN-enabled human activity recognition, and give some recommendations on how to select an appropriate method for a particular activity recognition application. Rama K. Yedavalli and Rohit K. Belapurkar summarize a number of important applications of WSNs for aircraft systems, such as distributed aircraft engine control, aircraft flight control, aircraft engine and structural health monitoring systems, along with a discussion on the technological challenges and future research directions.

The second category consists of 7 papers and is devoted to collaborative signal and information processing in WSNs which employs collaborative sensor resource management in the signal and information processing. Juo-Yu Lee et al. study novel approximate maximum likelihood (AML) algorithm for multisource direction-of-arrival (DOA) estimation using 3D acoustic sensor array. The paper also derives the Cramer-Rao bound (CRB) for the performance of the proposed AML algorithm. Yang Weng et al. consider the random field estimation in WSNs where the field can be described by unknown parameters to be estimated. To address the limited resources, a greedy sensor selection scheme is proposed according to the information gain of the sensors. A distributed algorithm is also developed by consensus based incremental sensor node selection. Bin Zhao et al. study the sensor selection problem in WSNs for received signal strength-based source localization. They find that due to the impacts of the network topology, the localization accuracy may not be always improved with the increasing of the number of selected sensors. Consequently they propose a sensor selection heuristic based on the model accuracy and the network geometry analysis. Yonggui Liu et al. present an energy-balanced multiple-sensor collaborative scheduling scheme for maneuvering target tracking in WSNs based on a distributed interactive multiple model (IMM) filter 
and adaptively changing the sampling intervals. Jian Xu et al. give the theoretic and experimental analysis for the quantized noise with quantized measurements and propose a corresponding state estimation algorithm. By applying the innovation analysis approach, Shuli Sun and Tian Tian study the optimal linear estimator problem for linear discrete-time stochastic systems with random measurement delays. Differently, Michael J. Walsh et al. study the hybrid RF based localization and QoS-based power control problem for WSNs, and test the proposed scheme experimentally on a fully compliant 802.15.4 test-bed.

The third category consists of 6 papers and is devoted to the networking protocols in WSNs. Xiaoling Zhang et al. develop a novel distributed and online joint routing, link scheduling and power control scheme for multichannel WSNs under the physical interference model to trade-off among energy consumption, end-to-end delay, and network throughput. Chaonong $\mathrm{Xu}$ et al. propose a new topology-transparent MAC scheduling algorithm based on a special balanced incomplete block design. The proposed protocol is independent of the network topology with good scalability. Meng Zheng et al. introduce the link replicator factor concept to stand for the number of reserved time slots of the link in each MAC superframe and study the optimal replicator factor control problem in TDMA-based MAC protocol in WSNs. Kai Ma et al. presents a Raiffa-Kalai-Smorodinsky bargaining solution (RBS) for cooperative bandwidth allocation in WSNs after partitioning the nodes into cooperative groups. Shujiang Li et al. propose an energy efficient multipath routing protocol for WSNs by considering the wireless interference. Jiguo Yu et al. present an energy-driven unequal clustering protocol (EDUC) for heterogeneous WSNs, together with an adaptive cluster head rotation scheme.

The guest editors hope that this special issue can provide a snapshot of the latest advances in WSNs and stimulate more research interest and efforts in WSN research and development.

The guest editors would like to express their sincere gratitude to Professor Frank L. Lewis, JCTA Deputy Editor-inChief, and Professor Daizhan Cheng, JCTA Editor-in-Chief, for their invaluable and consistent supports to this special issue. We would also like to thank all the reviewers for their professional contributions and Tiefeng Zou for her continuous help and patience.

\section{Guest editors:}

Wendong Xiao

Institute for Infocomm Research, Singapore

E-mail: wxiao@i2r.a-star.edu.sg

\section{Sajal K. Das}

The University of Texas at Arlington, U.S.A.

E-mail: das@uta.edu

\section{Haibin Yu}

Chinese Academy of Sciences, China

E-mail: yhb@ sia.cn

\section{Chen Khong Tham}

National University of Singapore, Singapore

E-mail: eletck@nus.edu.sg 